\title{
Effects of vestibular stimulation on gait stability and variability
}

\author{
Rina M. Magnani ${ }^{1 *}$, Jaap H. van Dieën ${ }^{2}$, Sjoerd M. Bruijn ${ }^{2,3}$ \\ ${ }^{1}$ Department of Physiotherapy, School of Physical Education and Physical Therapy, State University of Goiás, \\ Goiânia/GO, Brazil \\ ${ }^{2}$ Department of Human Movement Sciences, Faculty of Behavioral and Movement Sciences, Vrije Universiteit \\ Amsterdam, Amsterdam Movement Sciences, Amsterdam, The Netherlands \\ ${ }^{3}$ Institute Brain and Behavior Amsterdam, Amsterdam, The Netherlands \\ *Corresponding author: rinamagnani@gmail.com
}

\section{Abstract}

Vestibular information modulates muscle activity during gait, presumably to contribute stability, because noisy electrical vestibular stimulation perturbs gait stability. An important mechanism to stabilize gait in the mediolateral direction is to coordinate foot placement based on a sensory estimate of the trunk center of mass state, to which vestibular information appears to contribute. We, therefore expected that noisy vestibular stimulation would decrease the correlation between foot placement and trunk center of mass state. Moreover, as vestibular modulation of muscle activity during gait depends on step width, we expected stronger effects for narrow-base than normal walking, and smaller effects for wide-base walking. In eleven healthy subjects we measured the kinematics of the trunk (as a proxy of the center of mass), and feet, while they walked on a treadmill in six conditions, including three different step widths: control (preferred step width), narrow-base (steps smaller than hip width), and wide-base (with steps greater than hip width). The three conditions were conducted with and without a bipolar electrical stimulus, applied behind the ears (5 $\mathrm{mA})$. Walking with EVS reduced gait stability but increased the foot placement to center of mass correlation in different step width conditions. The narrow-base walking was the most stable condition and showed a stronger correlation between foot placement and center of mass state. We argue that EVS destabilized gait, but that this was partially compensated for by tightened control over foot placement, which would require successful use of other than vestibular sensory inputs, to estimate center of mass movement. 
Introduction

Electrical vestibular stimulation has been applied to probe the role of the human vestibular system during walking (Horslen et al. 2014). This has shown vestibular contributions to control of mediolateral stability during walking, in particular during ankle torque at push-off and foot placement at heel-strike (Jahn et al. 2000; Blouin et al. 2011; Dakin et al. 2011, 2013). Stochastic or unpredictable electrical vestibular stimulation provides erroneous input to the vestibular system and thus decreases gait stability (Sloot et al. 2011; van Schooten et al. 2011; Magnani et al. 2021) and increases trunk movement variability (van Schooten et al. 2011). In a recent study, we showed that the coupling of electrical vestibular stimulation to muscle activity and the resulting ground reaction forces was modulated as a function of stabilization demands (Magnani et al. 2021). We found that the mediolateral ground reaction force was less coupled to vestibular stimulation during externally stabilized walking than during normal walking. Moreover, when walking with narrower steps, a stronger coupling between ground reaction force and vestibular stimulation was present. Overall, these findings suggests that the importance of vestibular input for control of human walking is dependent on stability demands.

If indeed vestibular contributions to gait depend on stability demands, we expect the destabilizing effects of electrical vestibular stimulation (Sloot et al. 2011; van Schooten et al. 2011) to also depend on stability demands. For instance, as decreased coupling between electrical vestibular stimulation and ground reaction forces has been shown in wide-base walking, we hypothesized the decrease in stability due to vestibular stimulation during widebase walking to be less than during narrow-base walking. Conversely, since narrow-base walking has shown to increase coupling between electrical vestibular stimulation and ground reaction forces, we hypothesized a larger decrease in gait stability under the vestibular stimulation during narrow-base walking.

In the current study, we tested how stochastic vestibular stimulation affects gait stability and how it affects the regulation of gait stability compared to normal (non-stimulated) walking in different step width conditions. We measured the effect of electrical vestibular stimulation on gait stability by means of the local divergence exponent, a measure of local dynamic stability, as done in previous studies (Sloot et al. 2011; van Schooten et al. 2011). In addition, we assessed foot placement, which has been suggested to be the main mechanism to regulate 
mediolateral stability (Wang and Srinivasan 2014; Arvin et al. 2016). The mediolateral center of mass state or closely related pelvis or trunk states predict $>80 \%$ of the variance in mediolateral foot placement, and this measure of predictability of foot placement is gaining popularity as an index of gait stability or the control thereof (Rankin et al. 2014; Wang and Srinivasan 2014; Dean and Kautz 2015; Perry and Srinivasan 2017; Mahaki et al. 2019; Seethapathi and Srinivasan 2019; van Leeuwen et al. 2020). Vestibular stimulation during gait initiation was shown to affect foot placement, suggesting that it affects the estimate of the center of mass movement used for foot placement control (Reimann et al. 2017). We therefore hypothesized that random electrical vestibular stimulation would decrease the correlation between foot placement and trunk motion. Moreover, we hypothesized that, given the dependence of the contribution of vestibular information to mediolateral control, the decrease in correlation would be stronger in narrow-base walking than in normal walking and less strong in wide-base walking.

\section{Methods}

Ethics Statement and Participants We measured 23 healthy young adults between 24 and 33 years recruited from the university campus. Twelve participants were excluded during data analysis due to technical problems in the collection of electromyography $(n=3)$ or kinematic data $(n=9)$ in any of the trials recorded. Data of eleven healthy subjects (four female/seven male; mean age $28.8 \pm 3.1$ years) were included in the analysis. None of the participants reported any auditory, vestibular, neurologic, cardiovascular, or orthopedic disorders, and all had a normal or corrected-tonormal vision. The participants agreed to participate in the study by signing the informed consent form, and the study was approved by the Ethics Committee of the Faculty of Behavioural and Movement Sciences of the VU Amsterdam (VCWE-2017-158).

Instrumentation

Kinematic data were recorded using a 3D motion analysis system (Optotrak Northern Digital Inc., Waterloo, Ontario, Canada) operating at 100 samples per second. Light emitting diodes (LED) clusters were positioned at the occipital lobe, the spinous process of the sixth thoracic vertebra (T6), the posterior superior iliac spines and bilaterally at the calcaneus. Forces were 
collected from force plates embedded in the treadmill (Motekforce Link, The Netherlands) sampling at 200 samples per second.

Percutaneous bipolar electrical stimulation was used to modulate the firing rate of the vestibular nerves, using an isolated linear stimulator (STMISOLA, BIOPAC System Inc., Germany). The stimulation was applied by flexible carbon surface electrodes affixed with adhesive tape to the clean and dry skin of the subjects' mastoid processes, and further stabilized by an elastic band. The current varied randomly in the frequency band of 0 to 25 $\mathrm{Hz}$, with a peak amplitude of $5 \mathrm{~mA}$ and RMS of $~ 1.2 \mathrm{~mA}$.

Protocol

The protocol was conducted with subjects wearing their own shoes, walking on a level treadmill at fixed speed of $2.1 \mathrm{~km} / \mathrm{h}$ guided by the beat of a metronome at $78 \mathrm{steps} / \mathrm{min}$ for six conditions. Although vestibular stimulation was found to reduce gait stability at slow, preferred, and fast walking speeds (van Schooten et al. 2011), this walking speed was chosen to ensure clear vestibular system-driven balance responses, since slow walking relies more on vestibular information than fast walking (Dakin et al. 2013). In a similar way, a decrease in heading error was seen in vestibular patients and controls when running with electrical vestibular stimulation when compared to walking (Brandt 2000; Jahn et al. 2000). All in all, the literature suggests that fast walking relies less on vestibular information than slow walking.

The trials were characterized by step width, here called Condition (control, narrow base, wide base) and by the stimulus presence (EVS and no-EVS). For the control condition, subjects walked with their usual step width; during the narrow-base walking, subjects were asked to adopt a smaller base of support (smaller than hip width); for the wide-base walking subjects were requested to increase the base of support adopting step width greater than hip width. Trial order for the different condition was randomized for each subject and given a short 2 minute break between trials.

Data analysis

Gait events were identified from center of pressure data (Roerdink et al. 2008), and a complete gait cycle was defined by the time between heel contact and subsequent heel contact of the same limb. Since the minimum number of strides over conditions and subjects was 32 , we calculated all gait parameters for the last 32 strides of each data set. The mean and standard deviation of stride time (ST) (calculated as the duration between two 
consecutive heel contacts of the same lower limb) and step width (SW) (determined as the mediolateral distance between the heels during heelstrikes) were calculated. Gait stability was evaluated by the local divergence exponent calculated from the T6 mediolateral velocity time series (Dingwell and Cusumano 2000). To this end, the time series was first normalized to 3200 samples, after which a 5 dimensional state space (embedding delay = 10 samples) was constructed. Next, for each point in the state space, the divergence between each datapoint and its nearest neighbor was tracked over time. The mean of the log of these curves was then taken as the logarithmic rate of divergence and the local divergence exponent was calculated as the slope to this curve from 0-0.5 strides (Rosenstein et al. 1993; Stenum et al. 2014; Bruijn 2017).

The foot placement strategy was quantified using a regression equation predicting the subsequent mediolateral foot placement based on the position and velocity of mediolateral trunk center of mass at midswing of the preceding swing phase and at the end of the swing phase at heelstrike. The ratio of predicted foot placement variance to actual foot placement variance $\left(R^{2}\right)$ was used as outcome measures. Higher $R^{2}$ represents a smaller difference between predicted and real foot placements indicating high correlation between mediolateral trunk center of mass state and the subsequent mediolateral foot placement (Wang and Srinivasan 2014). The T6 marker was used to approximate the ML trunk center of mass (CoM) position. The ML trunk CoM velocity (VCoM) was calculated as the first derivative of the ML trunk CoM position time-series. We used the following regression equation:

$$
\mathbf{F P}=\boldsymbol{\beta 1}(\mathbf{i}) \cdot \operatorname{CoM}(\mathbf{i})+\boldsymbol{\beta 2}(\mathbf{i}) \cdot \operatorname{VCoM}(\mathbf{i})+\varepsilon(\mathbf{i})
$$

where $\beta 1$ and $\beta 2$ are the regression coefficients, $\varepsilon$ the error, and $i$ the indicator of the $\%$ of swing phase that was used for the prediction (Wang and Srinivasan 2014; Mahaki et al. 2019; Bruijn and Van Leeuwen 2020; van Leeuwen et al. 2020).

Statistical Analysis

The assumption of normality was checked by the Shapiro-Wilk test ( $p>0.05)$. The gait parameters, SW, ST and their variabilities, local divergence exponent and the $R^{2}$ of foot placement at the midswing and heelstrike phases, were compared by repeated measures analysis of variance with two factors, Stimulation (EVS - electrical vestibular stimulation and no-EVS) x Condition (control, narrow, wide) effects and the post hoc analysis was done using 
the Tukey's test. All statistical analyses were performed by Jamovi software (Version 0.9.2.8, Jamovi Org.), using $p<0.05$ as a threshold for significance.

Results

For all repeated measures ANOVA analyses, the results are presented in Table 1. As expected, step width showed Condition effects, where control walking had significantly wider step widths than narrow-base walking and narrower step width than wide-base walking. Step width also showed a significant Condition x EVS interaction, and no significant EVS effect, indicating that the effect of EVS was different for walking at different step widths. Post hoc testing showed that during EVS trials the step width in the control condition was significantly higher than in the narrow-base condition and lower than in the wide-base condition, the same was seen for the normal (no-EVS) trials. Step width variability showed a significant Condition $x$ EVS interaction, as well as a significant EVS effect. These effects indicated that overall, step width variability increased when walking with EVS, but not for the wide-base condition (Figure 1). Step width variability also showed a significant effect of Condition showing that control condition had a significantly lower step width variability than narrow-base walking.

Table 1: Statistical results. The bold text represents the statistically significant results $(p<0.05)$. SW: step width; ST: stride time; var: variability; LDE: local divergence exponent; R2: percentage of explained variance of foot placement; EVS: electrical vestibular stimulation.

\begin{tabular}{c|c|c|c} 
& EVS effects & Condition effects & EVS x Condition effects \\
\hline $\begin{array}{c}\text { SW } \\
(m)\end{array}$ & $F(1,10)=1.50 ; p=0.248 ; n^{2}=0.002$ & $F(2,20)=53.28 ; p<0.001 ; n^{2}=0.618$ & $F(2,20)=8.44 ; p=0.002 ; n^{2}=0.007$ \\
\hline $\begin{array}{c}\text { SW var } \\
(m)\end{array}$ & $F(1,10)=10.83 ; p=0.008 ; n^{2}=0.105$ & $F(2,20)=6.17 ; p=0.008 ; n^{2}=0.129$ & $F(2,20)=3.86 ; p=0.038 ; n^{2}=0.039$ \\
\hline $\begin{array}{c}\text { ST } \\
(s)\end{array}$ & $F(1,10)=3.40 ; p=0.095 ; n^{2}=0.033$ & $F(2,20)=5.62 ; p=0.012 ; n^{2}=0.145$ & $F(2,20)=2.26 ; p=0.130 ; n^{2}=0.145$ \\
\hline $\begin{array}{c}S T \text { var } \\
(s)\end{array}$ & $F(1,10)=9.17 ; p=0.013 ; n^{2}=0.140$ & $F(2,20)=2.74 ; p=0.088 ; n^{2}=0.029$ & $F(2,20)=0.31 ; p=0.730 ; n^{2}=0.006$ \\
\hline $\begin{array}{c}\text { LDE } \\
\begin{array}{c}R^{2}- \\
\text { midswing }\end{array}\end{array}$ & $F(1,10)=7.47 ; p=0.021 ; n^{2}=0.140$ & $F(2,20)=11.12 ; p<0.001 ; n^{2}=0.135$ & $F(2,20)=0.238 ; p=0.791 ; n^{2}=0.003$ \\
\hline $\begin{array}{c}R^{2}- \\
\text { heelstrike }\end{array}$ & $F(1,10)=2.95 ; p=0.117 ; n^{2}=0.027$ & $F(2,20)=9.73 ; p=0.001 ; n^{2}=0.235$ & $F(2,20)=5.18 ; p=0.063 ; n^{2}=0.030$ \\
\hline
\end{tabular}



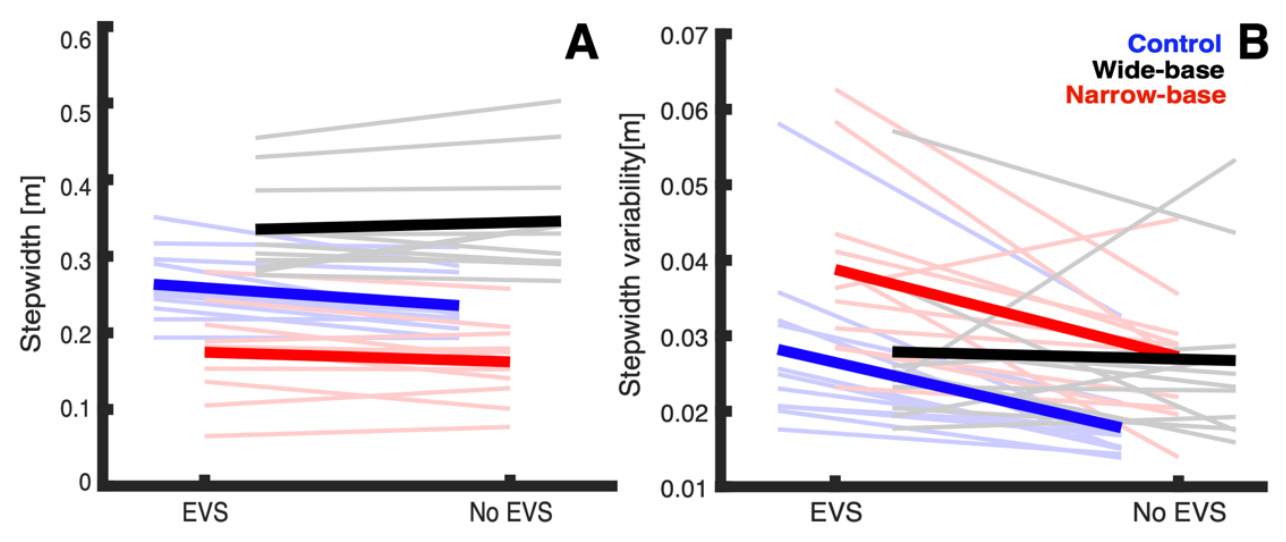

Figure 1: Mean values of step width (A) and step width variability (B). Thick lines represent mean values and thin lines show individual data for each condition according to the following colors - blue lines: control condition; black lines: wide-base condition; red lines: narrow-base condition.

Stride time showed a significant Condition effect, indicating that during narrow-base walking, subjects showed longer stride time than during control walking condition (Figure 2). Stride time variability was significantly higher during EVS trials than during no-EVS trials, presenting no significant effects of Condition nor an interaction.
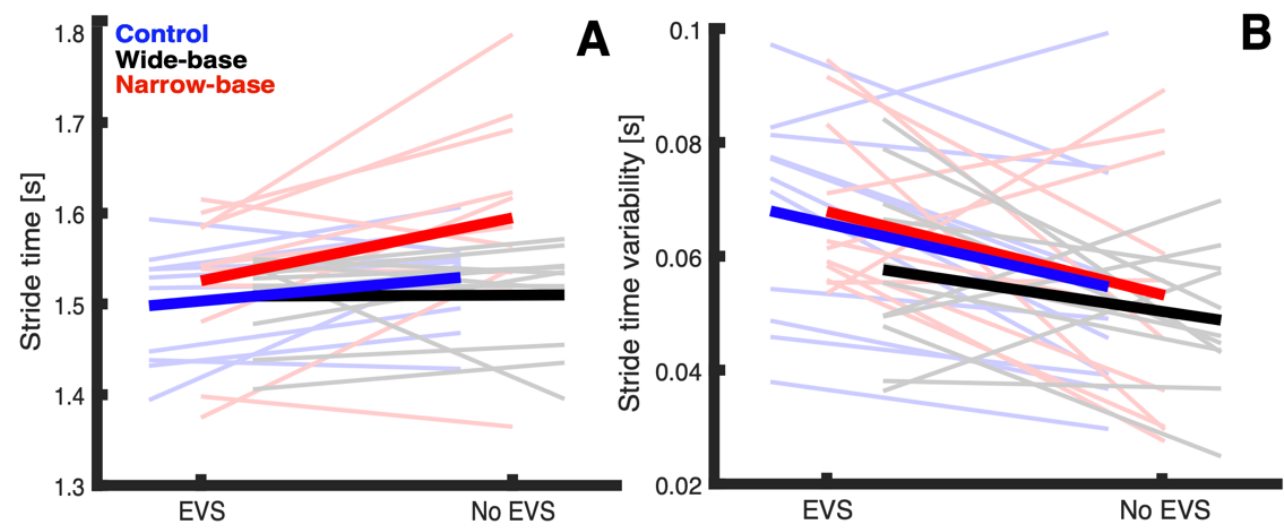

Figure 2: Mean values of stride time (A) and stride time variability (B). Thick lines represent mean values and thin lines show individual data for each condition according to the following colors - blue lines: control condition; black lines: wide-base condition; red lines: narrow-base condition.

Local dynamic stability was significantly decreased (i.e. increased LDE values) during EVS conditions. Moreover, there was a significant effect of Condition on LDE, where the post hoc 
tests indicated that wide-base walking was less stable than the control condition. In contrast with our hypothesis, there was no significant interaction effect on LDE (Figure 3).

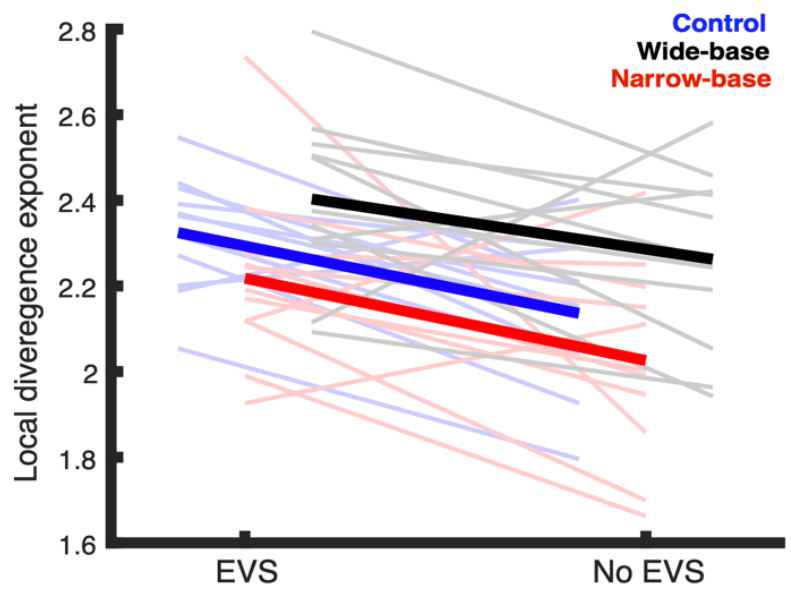

Figure 3: Mean values of mediolateral local divergence exponent. Thick lines represent mean values and thin lines show individual data for each condition according to the following colors - blue lines: control condition; black lines: wide-base condition; red lines: narrow-base condition.

Contrary to our expectations, foot placement was more tightly coordinated to trunk motions during EVS walking, as the $\mathrm{R}^{2}$ in the midswing phase showed significantly higher values during EVS, with no effects of Condition nor an interaction. For the $\mathrm{R}^{2}$ at heelstrike, this effect of EVS was not found. However, the $\mathrm{R}^{2}$ at heelstrike showed a significant Condition effect, with the control condition having a lower percentage of explained variance compared to narrow-base walking (Figure 4).

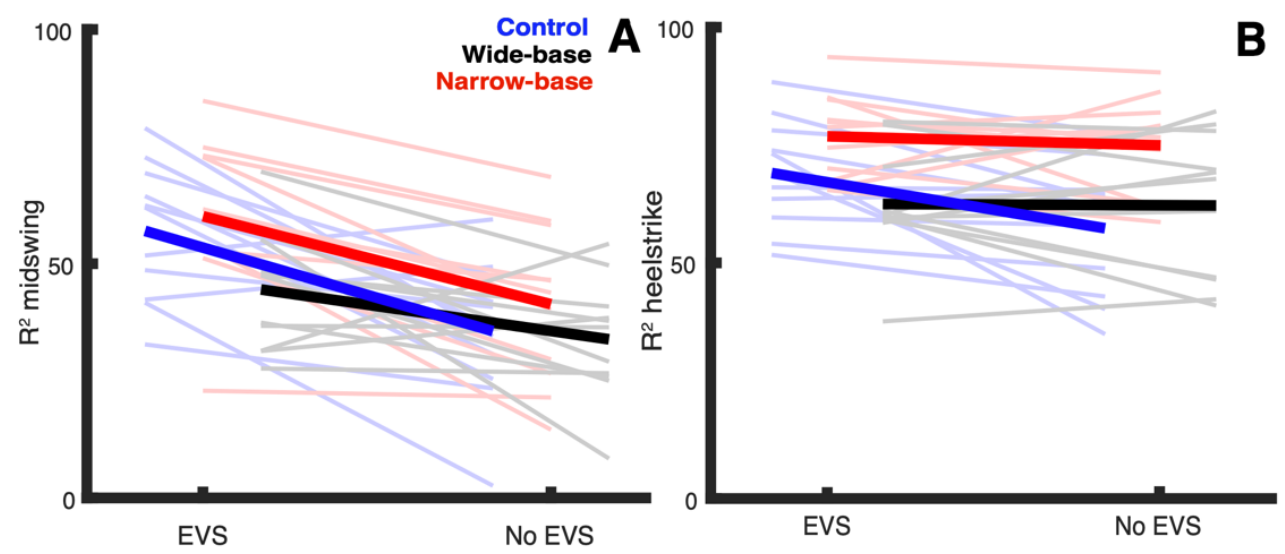

Figure 4: Mean values of $R^{2}$ (percentage of explained variance) at midswing (A) and at heelstrike (B). Thick lines represent mean values and thin lines show individual data for each condition according to the following colors - blue lines: control condition; black lines: wide-base condition; red lines: narrowbase condition. 
Discussion

This study aimed to test the effects of stochastic EVS on gait stability and its regulation through foot placement in walking with different step widths. As we had previously shown a decreased coupling between EVS and ground reaction forces in wide-base walking compared to normal walking, we expected that EVS during wide-base walking would have less effect on gait stability and its regulation than during normal walking. Likewise, as we have shown increased coupling between EVS and ground reaction forces in narrow-base walking, we hypothesized a larger effect of EVS on gait stability and its regulation during narrow-base walking than during normal walking.

Walking with EVS led to reduced gait stability (as evidenced by a higher LDE), as found previously (Sloot et al. 2011; van Schooten et al. 2011; Magnani et al. 2021) during all conditions of step width. For the no-EVS conditions, wide-base walking was less stable, while narrow-base walking was most stable. The same pattern was seen during the EVS walking trials, as reported before (Young and Dingwell 2012; Magnani et al. 2021).

The correlation between foot placement and trunk CoM state during midswing, as a reflection of the accuracy of foot placement for the regulation of mediolateral gait stability, was greater under EVS, for all base of support conditions, contradicting our hypothesis. At heelstrike, the correlation between foot placement and CoM state was also higher when walking with EVS for the control and narrow-base conditions, although not significantly so. During narrow-base walking the contribution of the vestibular system seems to be large for the erector spinae muscles activity (Magnani et al. 2021), we expected that EVS would perturb estimation of trunk movement and thus lead to a lower $\mathrm{R}^{2}$.

Our unexpected finding of higher (or non-changed) correlations between foot placement and trunk CoM state and the opposite effect of decreased gait stability (increased local divergence exponent values) during EVS walking may be surprising, as foot placement is thought of as one of the main ways to control gait stability. However, it should be kept in mind that foot placement is only one mechanism by which stability can be controlled. Other mechanisms include the control of the center of pressure (Reimann et al. 2018; van Leeuwen et al. 2021), and angular momentum control (Hof 2008). Hence, it may well be that the improved foot 
placement control during walking with EVS was negated by a decrease in other control mechanisms, leading to a net reduction in gait stability.

This then leads to the question what could have caused the increase in correlation between trunk kinematic state and foot placement during EVS walking found here? We propose three potential mechanisms. The first is that the vestibular system is mostly involved in stance leg control. The vestibular input might give meaningful information for control of stance leg muscle, since it was shown the coupling is related to horizontal forces (Magnani et al. 2021). However, such a decrease in stance leg control would most likely also result in a decrease in foot placement control (van Leeuwen et al. 2021), and hence, isn't the most likely explanation. Moreover, EVS was previously shown to affect foot placement during gait initiation (Reimann et al. 2017).

The second potential explanation is stochastic resonance. Stochastic resonance is defined as an increase of the detectability of subthreshold signals in non-linear systems, such as the vestibular system, when low-level noise input is applied (Mulavara et al. 2015; Iwasaki et al. 2018; Stefani et al. 2020). Stochastic resonance has shown positive effects improving ocular stabilization reflexes in response to whole-body tilt, standing posture and gait stability in impaired, elderly and healthy subjects (Dakin 2007; Mulavara et al. 2011, 2015; Dilda et al. 2014; Iwasaki et al. 2014, 2018; Moore et al. 2015; Wuehr et al. 2016). However, given that we found a decrease in gait stability alongside an increase in foot placement control when walking with EVS, stochastic resonance is not a very likely mechanism (unless it would work only for foot placement, and not for stance leg control).

The third potential explanation involves the idea of sensory reweighting. In case of noise in, or absence of one sensory input, other sensory system are more reliable and become more heavily weighted by the central nervous system (Peterka et al. 2002; Cenciarini and Peterka 2006; Jeka et al. 2008, 2010; Assländer and Peterka 2014; Reimann et al. 2018). Vestibular stimulation may cause down-weighting of vestibular information, increasing reliance of foot placement on visual and/or proprioceptive inputs, which have both been shown to potentially affect foot placement coordination in gait (Arvin et al. 2018; Reimann et al. 2018; Fettrow et al. 2019). It is however unclear why this would improve foot placement relative to the no-EVS condition, while at the same time (most likely) reducing stance leg control. Potentially the challenge experienced due to EVS elicits a tighter control of foot placement, which would then be only partially successful in maintaining gait stability. This would also explain why 
narrow-base walking showed more tight control of foot placement. To test if indeed sensory reweighting plays a role, we would need to test correlations between foot placement and CoM state in response to the visual or proprioceptive perturbations during EVS.

Regarding the effects of step width Conditions, we previously showed an increased coupling of EVS to ground reaction forces during narrow-base walking (as compared to control walking), which we took to suggest that the contribution of vestibular input to control of gait is larger during narrow-base walking (Magnani et al. 2021). The current findings do not support this idea. Narrow-base walking showed a higher percentage of explained variance of foot placement $\left(R^{2}\right)$ for both stimulus conditions. In contrast, during the wide-base walking we found a lower percentage of explained variance compared to the control condition. These effects of step width instructions are in line with findings by (Perry and Srinivasan 2017), who also showed that control of foot placement is tighter during narrow-base walking. This might be due to the fact that the increased base of support during wide-base walking increases margins of safety, so it would need less foot placement modulations (Perry and Srinivasan 2017; van Leeuwen et al. 2020).

In conclusion, stochastic electrical vestibular stimulation decreases local dynamic stability of gait in different step width conditions, but foot placement was more correlated with trunk motion during walking with electrical vestibular stimulation. Most likely, the destabilized gait under EVS was partially compensated for by tightened control over foot placement, which would require successful use of other than vestibular sensory inputs, to estimate center of mass movement.

\section{Declarations}

\section{Funding}

RMM was funded by CAPES (PDSE 19/2016). SMB was funded by a grant from the Netherlands Organization for Scientific Research (016.Vidi.178.014).

Conflicts of interest

The authors declare that they have no competing interests.

\section{Availability of data and material (data transparency)}

The data and code for this manuscript can be found at https://surfdrive.surf.nl/files/index.php/s/dUu3uRQyVHIN2g3 and will be published to Zenodo upon acceptance. 
References

Arvin M, Hoozemans MJM, Pijnappels M, et al (2018) Where to Step ? Contributions of

Stance Leg Muscle Spindle Afference to Planning of Mediolateral Foot Placement for Balance Control in Young and Old Adults. 9:1-10.

https://doi.org/10.3389/fphys.2018.01134

Arvin M, Mazaheri M, Hoozemans MJM, et al (2016) Effects of narrow base gait on mediolateral balance control in young and older adults. J Biomech 49:1264-1267. https://doi.org/10.1016/j.jbiomech.2016.03.011

Assländer L, Peterka RJ (2014) Sensory reweighting dynamics in human postural control. J Neurophysiol 111:1852-1864. https://doi.org/10.1152/jn.00669.2013

Blouin J, Dakin CJ, Doel K Van Den, et al (2011) Extracting phase-dependent human vestibular reflexes during locomotion using both time and frequency correlation approaches. 1484-1490. https://doi.org/10.1152/japplphysiol.00621.2011.

Brandt T (2000) Vestibulopathic gait: you're better off running than walking. Curr Opin Neurol 13:3-5. https://doi.org/10.1097/00019052-200002000-00002

Bruijn SM (2017) Local Dynamic Stability.

https://zenodo.org/record/1039387\#.YR6k2y2708Q

Bruijn SM, Van Leeuwen AM (2020) FootPlacement: Initial Release.

http://doi.org/10.5281/zenodo.4292212

Cenciarini M, Peterka RJ (2006) Stimulus-Dependent Changes in the Vestibular Contribution to Human Postural Control. J Neurophysiol 95:2733-2750.

https://doi.org/10.1152/jn.00856.2004

Dakin CJ (2007) The Development of Stochastic Vestibular Stimulation and its Application to Dynamic Vestibular Evoked Responses

Dakin CJ, Inglis JT, Blouin JS (2011) Short and medium latency muscle responses evoked by electrical vestibular stimulation are a composite of all stimulus frequencies. Exp Brain Res 209:345-354. https://doi.org/10.1007/s00221-011-2549-7

Dakin CJ, Inglis JT, Chua R, Blouin J (2013) Muscle-specific modulation of vestibular reflexes with increased locomotor velocity and cadence. J Neurophysiol 110:86-94. https://doi.org/10.1152/jn.00843.2012

Dean JC, Kautz SA (2015) Foot placement control and gait instability among people with stroke. J Rehabil Res Dev 52:577-590. https://doi.org/10.1682/JRRD.2014.09.0207 
Dilda V, Morris TR, Yungher DA, et al (2014) Central adaptation to repeated galvanic vestibular stimulation: Implications for pre-flight astronaut training. PLoS One 9:. https://doi.org/10.1371/journal.pone.0112131

Dingwell JB, Cusumano JP (2000) Nonlinear time series analysis of normal and pathological human walking. Chaos 10:848-863. https://doi.org/10.1063/1.1324008

Fettrow T, Reimann H, Grenet D, et al (2019) Interdependence of balance mechanisms during bipedal locomotion. PLoS One 14:1-15. https://doi.org/10.1371/journal.pone.0225902

Hof AL (2008) The 'extrapolated center of mass' concept suggests a simple control of balance in walking. Hum Mov Sci 27:112-125. https://doi.org/10.1016/j.humov.2007.08.003

Horslen BC, Dakin CJ, Inglis JT, et al (2014) Modulation of human vestibular reflexes with increased postural threat. J Physiol 592:3671-3685. https://doi.org/10.1113/jphysiol.2014.270744

Iwasaki S, Fujimoto C, Egami N, et al (2018) Noisy vestibular stimulation increases gait speed in normals and in bilateral vestibulopathy. Brain Stimul 11:709-715. https://doi.org/10.1016/j.brs.2018.03.005

Iwasaki S, Yamamoto Y, Togo F, et al (2014) Noisy vestibular stimulation improves body balance in bilateral vestibulopathy. Neurology 82:969-975. https://doi.org/10.1212/WNL.0000000000000215

Jahn K, Strupp M, Schneider E, et al (2000) Differential effects of vestibular stimulation on walking and running. Neuroreport 11:1745-8

Jeka JJ, Allison LK, Kiemel T (2010) The Dynamics of Visual Reweighting in Healthy and FallProne Older Adults. J Mot Behav 42:197-208. https://doi.org/10.1080/00222895.2010.481693

Jeka JJ, Oie KS, Kiemel T (2008) Asymmetric adaptation with functional advantage in human sensorimotor control. Exp Brain Res 191:453-463. https://doi.org/10.1007/s00221008-1539-x

Magnani RM, Bruijn SM, van Dieën JH, Forbes PA (2021) Stabilization demands of walking modulate the vestibular contributions to gait. bioRxiv Prepr 1-23. https://doi.org/https://doi.org/10.1101/2020.09.30.319434

Mahaki M, Bruijn SM, Van Dieën JH (2019) The effect of external lateral stabilization on the 
use of foot placement to control mediolateral stability in walking and running. PeerJ 2019:. https://doi.org/10.7717/peerj.7939

Moore ST, Dilda V, Morris TR, et al (2015) Pre-adaptation to noisy Galvanic vestibular stimulation is associated with enhanced sensorimotor performance in novel vestibular environments. Front Syst Neurosci 9:88. https://doi.org/10.3389/fnsys.2015.00088 Mulavara AP, Fiedler MJ, Kofman IS, et al (2011) Improving balance function using vestibular stochastic resonance: Optimizing stimulus characteristics. Exp Brain Res 210:303-312. https://doi.org/10.1007/s00221-011-2633-z

Mulavara AP, Kofman IS, Dios YE De, et al (2015) Using low levels of stochastic vestibular stimulation to improve locomotor stability. Front Syst Neurosci 9:

Perry JA, Srinivasan M (2017) Walking with wider steps changes foot placement control , increases kinematic variability and does not improve linear stability

Peterka RJ, Goodworth AD, Mellodge P, et al (2002) Sensorimotor Integration in Human Postural Control. J Neurophysiol 1097-1118

Rankin BL, Buffo SK, Dean JC (2014) A neuromechanical strategy for mediolateral foot placement in walking humans. 2:374-383. https://doi.org/10.1152/jn.00138.2014

Reimann H, Fettrow T, Jeka JJ (2018) Strategies for the Control of Balance During Locomotion. Kinesiol Rev 7:18-25. https://doi.org/10.1123/kr.2017-0053

Reimann H, Fettrow TD, Thompson ED, et al (2017) Complementary mechanisms for upright balance during walking. 1-16. https://doi.org/10.5061/dryad.j0440

Roerdink M, Coolen BH, Clairbois BHE, et al (2008) Online gait event detection using a large force platform embedded in a treadmill. J Biomech 41:2628-32.

https://doi.org/10.1016/j.jbiomech.2008.06.023

Rosenstein MT, Collins JJ, Luca CJ De (1993) A practical method for calculating largest Lyapunov from small data sets. Phys D Nonlinear Phenom 65:117-134

Seethapathi N, Srinivasan M (2019) Step-to-step variations in human running reveal how humans run without falling. Elife 8:1-24. https://doi.org/10.7554/eLife.38371

Sloot LH, Van Schooten KS, Bruijn SM, et al (2011) Sensitivity of local dynamic stability of over-ground walking to balance impairment due to galvanic vestibular stimulation. Ann Biomed Eng 39:1563-1569. https://doi.org/10.1007/s10439-010-0240-y

Stefani SP, Serrador JM, Breen PP, Camp AJ (2020) Impact of galvanic vestibular stimulationinduced stochastic resonance on the output of the vestibular system: A systematic 
review. Brain Stimul 13:533-535. https://doi.org/10.1016/j.brs.2020.01.006

Stenum J, Bruijn SM, Jensen BR (2014) The effect of walking speed on local dynamic stability is sensitive to calculation methods. J Biomech 47:3776-3779.

https://doi.org/10.1016/j.jbiomech.2014.09.020

van Leeuwen AM, van Dieën JH, Daffertshofer A, Bruijn SM (2020) Step width and frequency to modulate: Active foot placement control ensures stable gait. bioRxiv 1-36.

https://doi.org/10.1101/2020.06.10.143867

van Leeuwen AM, van Dieën JH, Daffertshofer A, Bruijn SM (2021) Ankle muscles activate: mediolateral center of pressure control ensures stable gait. bioRxiv 2021.03.31.437904 van Schooten KS, Sloot LH, Bruijn SM, et al (2011) Sensitivity of trunk variability and stability measures to balance impairments induced by galvanic vestibular stimulation during gait. Gait Posture 33:656-660. https://doi.org/10.1016/j.gaitpost.2011.02.017

Wang Y, Srinivasan M (2014) Stepping in the direction of the fall: the next foot placement can be predicted from current upper body state in steady-state walking. Biol Lett 10:20140405. https://doi.org/10.1098/rsbl.2014.0405

Wuehr M, Nusser E, Krafczyk S, et al (2016) Noise-Enhanced Vestibular Input Improves Dynamic Walking Stability in Healthy Subjects. Brain Stimul 9:109-116. https://doi.org/10.1016/j.brs.2015.08.017

Young PMM, Dingwell JB (2012) Voluntarily changing step length or step width affects dynamic stability of human walking. Gait Posture 35:472-477. https://doi.org/10.1016/j.pestbp.2011.02.012.Investigations 